\title{
Control of entanglement in Ising-type networks with one and two excitations
}

\author{
J Novotný ${ }^{1}$, M Štefaňák ${ }^{1}$, T Kiss $^{2}$ and I Jex ${ }^{1}$ \\ ${ }^{1}$ Department of Physics, FJFI ČVUT, Břehová 7, 11519 Praha 1, Staré Město, Czech Republic \\ 2 Department of Nonlinear and Quantum Optics, Research Institute for Solid State Physics and \\ Optics, Hungarian Academy of Sciences, Konkoly-Thege u. 29-33, H-1121 Budapest, Hungary
}

Received 11 May 2005, in final form 6 September 2005

Published 28 September 2005

Online at stacks.iop.org/JPhysA/38/9087

\begin{abstract}
We analyse the dynamics of single- and two-particle states in Ising-type networks. The mutual entanglement is quantified using the concept of concurrence. We derive explicit expressions for the concurrence for singleand two-particle initial states in arbitrary passive networks and specify the result for Ising-type networks. We show how to design a network to prepare a prescribed pattern of entanglement for one excitation and study the maximum attainable entanglement for passive optical networks in general. The effect of network randomization on the average entanglement is also studied.
\end{abstract}

PACS numbers: 42.50.-p, 42.50.Ar, 03.65.Ud

\section{Introduction}

Linear optical instruments are particularly simple and versatile. They include beam splitters [1], phase shifters and parametric amplifiers. All of them are characterized by simple (linear) relations between the input and output fields. The creation operator of the outputs are given as linear combinations of input creation and annihilation operators. The coefficients of the linear transform characterize the physical properties of the devices and determine the characteristics of the output once the input is specified.

The linear elements represent interesting devices in their own right. They can be used to demonstrate striking quantum effects like destructive interference (on the beam splitter) which has quite a number of interesting applications ranging from simple beam splitting to quantum state identification and comparison [2]. The other large area of applications is connected with larger systems formed out of beam splitters and phase shifters. In this way, we obtain interferometers with a broad field of applications. Such arrangements of passive optical elements (networks) can be used for many purposes. They can serve naturally as real interferometers and can be applied to many practical applications such as many-state comparison, quantum state detection or photon number identification. 
Another possibility to employ interferometers is their use for implementing quantum computers. It has been shown how elementary quantum computation operations could be implemented using linear optics. While this direction might not be the most efficient one it can definitely serve as a way of demonstrating quite a number of quantum computation tasks on the principal-fundamental level.

A further direction of applications covers the possibility to mimic with networks the functioning of other systems. It has been shown for instance that the nearest-neighbour interaction which is extensively used in solid-state physics and statistical physics can be mimicked using arrays of balanced beam splitters. Starting from this particular system various interesting effects can be studied. For instance random walks in a quantum outfit can be studied on such systems with a number of implications for quantum information theory [3]. In addition, effects like localization or studies of correlation lengths among excitations could be studied on Ising-like networks [4-6]. Ising-type networks are extensively studied. The name stems from the formal analogy between the Ising model of statistical mechanics and the optical networks as discussed in detail by Törmä [7].

In the present paper we focus on the study of quantum interference effects in passive optical networks for single- and two-photon inputs. We focus primarily on the analysis of the behaviour of entanglement between the output modes of the network. We also study the inverse problem, i.e. to determine what type of bipartite entanglement structure can be prepared by a passive network. We specify the general results for a particular case of Ising-type network with balanced beam splitters. We show how random phase errors affect the total amount of entanglement. We point out that uniform phase errors leading to localization will considerably decrease entanglement.

The paper is organized as follows. In section 2, we introduce in some detail the Isingtype network, then we analyse the propagation of a single excitation in an arbitrary passive network and the entanglement properties of the states. We derive the condition under which the bipartite entanglement structure can be prepared by a passive network. Then we analyse the optimal value of entanglement in passive networks. We specify the obtained general results for the particular case of Ising-type network with balanced beam splitters and analyse the effects of phase randomization on the average entanglement. The entanglement properties of two excitations in passive networks are analysed in section 4. The conclusions summarize the obtained results.

\section{Ising-type networks}

One of the advantages of optical networks is the simplicity of its mathematical description. The basic element of the whole network is the beam splitter described by the transfer matrix $A$ having the form

$$
A=\left(\begin{array}{rr}
\cos \theta & \sin \theta \\
-\sin \theta & \cos \theta
\end{array}\right)
$$

The angle $\theta$ determines the transmissivity and reflectivity of the beam splitter. The network with nearest neighbour coupling is now formed by repeating a sequence of beam splitters with different transmissivities specified by angles $\theta, \phi$ as illustrated in figure 1 . The transfer matrix $U$ is determined as a product of two block diagonal matrices 


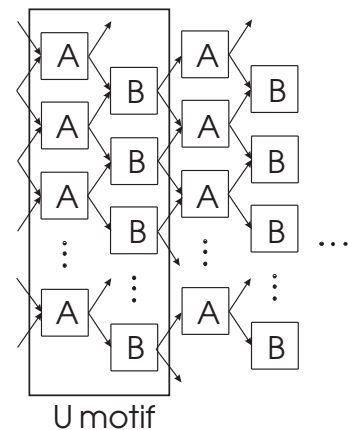

Figure 1. Ising-type network with two types of transformations repeated successively. The two layers of beam splitters of types A and B form the motif $U$.

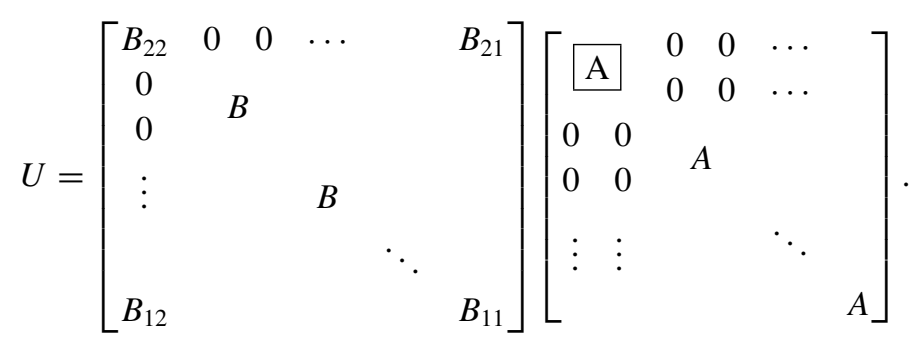

The matrix $B$ is defined as

$$
B=\left(\begin{array}{rr}
\cos \phi & \sin \phi \\
-\sin \phi & \cos \phi
\end{array}\right)
$$

Defining the network transform we used periodic boundary condition. This fact will not play any role in the following considerations. The action of the whole network on the input creation operators is defined by the application of the product of the two matrices

$$
W(M)=U^{M} \text {. }
$$

The matrix $U$ will be called the motif (see figure 1), the application of one of the block diagonal matrices will represent one layer. The properties of the network are determined by the eigenvalues of the matrix $U$. It was shown [7,8] that the eigenvalues $\exp \left( \pm \mathrm{i} \lambda_{n}\right)$ of the matrix $U$ can be determined from the equation

$$
\cos \lambda_{n}=\cos \theta \cos \phi+\cos \left(\frac{2 \pi n}{N}\right) \sin \theta \sin \phi,
$$

where $N$ is the number of beam splitters in one layer. For balanced beam splitters $\phi=\theta=\pi / 4$, which we will consider in the following, equation (5) reduces to

$$
\cos \lambda_{n}=\frac{1}{2}\left[1+\cos \left(\frac{2 \pi n}{N}\right)\right] .
$$

Using these eigenvalues many of the simpler properties of finite networks (finite number of beam splitters in one layer) like recurrences in the mode population for a single-photon input can be understood. Let us now analyse in some detail the propagation of a single photon in such passive networks. While the propagation of the photon number density was studied in great detail and became known as the quantum random walk not too much interest was paid to the evolution of entanglement in these cases. The basic difference in this respect is that the actual single-photon distribution across the output modes differs significantly from 
the classical random walk analogue. The maximum of the probability is not centred close to the input channel but is located at the outer wings of the distribution. The width of the distribution in the quantum case is much larger than in the classical case. The variance of the probability distribution grows quadratically with time (i.e., motifs passed), in contrast with the linear dependence for the classical random walk.

\section{Single-photon entanglement distribution in passive networks}

The unusual propagation of the probability distribution of a single-particle state in passive networks (including Ising-type networks as a special case) is closely linked to the distribution of entanglement in these networks [9, 10]. Entanglement refers to the quantum correlations between the particular output modes. We demonstrate this fact for a general one-particle state and a general passive network. We consider a general passive network characterized by the unitary transformation matrix $U$ between the input and the output mode creation operators

$$
\left(\begin{array}{c}
b_{1}^{\dagger} \\
b_{2}^{\dagger} \\
\vdots \\
b_{N}^{\dagger}
\end{array}\right)=U\left(\begin{array}{c}
a_{1}^{\dagger} \\
a_{2}^{\dagger} \\
\vdots \\
a_{N}^{\dagger}
\end{array}\right) ; \quad U=\left(\begin{array}{cccc}
U_{11} & U_{12} & \ldots & U_{1 N} \\
U_{21} & U_{22} & \ldots & U_{2 N} \\
\vdots & \vdots & \ddots & \vdots \\
U_{N 1} & U_{N 2} & \ldots & U_{N N}
\end{array}\right) .
$$

Let us choose an arbitrary one-particle input state

$$
\left|\psi_{\text {in }}\right\rangle=\sum_{i=1}^{N} \alpha_{i}\left|1_{i}\right\rangle=\sum_{i=1}^{N} \alpha_{i} a_{i}^{\dagger}|0\rangle, \quad \sum_{i=1}^{N}\left|\alpha_{i}\right|^{2}=1
$$

where the state $\left|1_{i}\right\rangle$ denotes state with zeros in all modes, except the $i$ th mode with a single photon (excitation). Then the output state has form

$\left|\psi_{\text {out }}\right\rangle=\mathcal{U}\left|\psi_{\text {in }}\right\rangle=\sum_{i=1}^{N} \alpha_{i} \mathcal{U} a_{i}^{\dagger}|0\rangle=\sum_{i=1}^{N} \alpha_{i} \mathcal{U} a_{i}^{\dagger} \mathcal{U}^{\dagger} \mathcal{U}|0\rangle=\sum_{k=1}^{N}\left(\sum_{i=1}^{N} U_{k i} \alpha_{i}\right)\left|1_{k}\right\rangle$,

where $\mathcal{U}$ denotes the propagator of the network. The probability to find a photon in the $n$th output mode is

$$
P(n)=\left|\left\langle 1_{n} \mid \psi_{\text {out }}\right\rangle\right|^{2}=\left|\sum_{i=1}^{N} U_{n i} \alpha_{i}\right|^{2}
$$

In the following we will investigate the evolution of two-particle quantum correlations.

For the bipartite entanglement of two-level systems we have a well-defined measure- - the concurrence $C(\rho)[11,12]$. Let us recall the definition of the concurrence for a bipartite system described by the density operator $\rho$. We denote by $\lambda_{1}, \lambda_{2}, \lambda_{3}, \lambda_{4}$ the decreasing sequence of eigenvalues of the associated operator

$$
R=\rho\left(\sigma_{y} \otimes \sigma_{y}\right) \rho^{*}\left(\sigma_{y} \otimes \sigma_{y}\right),
$$

where $\sigma_{y}$ is the Pauli matrix and a star $\left(^{*}\right)$ denotes the complex conjugation. Then the concurrence is defined as

$$
C(\rho)=\max \left\{0, \sqrt{\lambda_{1}}-\sqrt{\lambda_{2}}-\sqrt{\lambda_{3}}-\sqrt{\lambda_{4}}\right\} .
$$

Nonzero values of $C(\rho)$ indicate the presence of quantum correlations. For maximally entangled systems the concurrence attains the value 1 . 
To proceed we need to calculate the entanglement between two chosen arbitrary modes $i, j$ of the output state $\left|\psi_{\text {out }}\right\rangle$. At first we have to determine the density operator of the two particular modes

$$
\begin{aligned}
\rho_{i j} & =\operatorname{Tr}_{\text {rest } \neq i, j}\left(\left|\psi_{\text {out }}\right\rangle\left\langle\psi_{\text {out }}\right|\right) \\
& =\left(\begin{array}{cccc}
0 & 0 & 0 & 0 \\
0 & \left|\sum_{k} U_{i k} \alpha_{k}\right|^{2} & \left(\sum_{k} U_{i k} \alpha_{k}\right)\left(\sum_{k} U_{j k} \alpha_{k}\right)^{*} & 0 \\
0 & \left(\sum_{k} U_{i k} \alpha_{k}\right)^{*}\left(\sum_{k} U_{j k} \alpha_{k}\right) & \left|\sum_{k} U_{j k} \alpha_{k}\right|^{2} & 0 \\
0 & 0 & 0 & \sum_{l \neq i, j}\left|\sum_{k} U_{l k} \alpha_{k}\right|^{2}
\end{array}\right) .
\end{aligned}
$$

The simple form of the matrix (13) gives us the possibility to express analytically the eigenvalues of the reduced density operator $R\left(\rho_{i j}\right)$. The only nonzero eigenvalue is $\lambda_{1}=4\left|\sum_{k} U_{i k} \alpha_{k}\right|^{2}\left|\sum_{k} U_{j k} \alpha_{k}\right|^{2}$ and hence the concurrence reads

$$
C\left(\rho_{i j}\right)=2\left|\sum_{k} U_{i k} \alpha_{k}\right|\left|\sum_{k} U_{j k} \alpha_{k}\right| .
$$

Due to the equation for the photon number distribution (10) we get

$$
C\left(\rho_{i j}\right)=2 \sqrt{P(i) P(j)} .
$$

Let us emphasize that the obtained result is completely independent of the chosen type of the passive network and is valid for all input one-particle states. The structure of the bipartite entanglement of the output state has taken a simple form. All modes with nonzero probability $P(n)$ are mutually entangled. The remaining unpopulated modes stay unentangled. The degree of entanglement is independent of the relative phases between the populated modes.

\subsection{Controlling entanglement in passive networks with one excitation}

In the previous section, we have settled the question how the entanglement depends on the prescribed form of the passive network. Next, we will reverse the task. We will determine what type of the bipartite entanglement structure can be prepared by passive network and how to design an appropriate passive network. We formulate this problem in terms of concurrence.

From the previous section we know that only such entanglement structures can be realized in which populated modes are entangled with each other. Without loss of generality, suppose we have the first $n$ (where $n \leqslant N$ ) modes populated with a prescribed set of concurrences $C_{i j}$ $\left(C_{i j} \neq 0\right)$ for each pair of modes $i$ and $j$. Using $C_{i j}$ we will always assume that $i \neq j$ (note that $C_{i j}=C_{j i}$ ). Generally, all states with one excitation generated by a passive network have the form

$$
|\psi\rangle=\sum_{i=1}^{n} \lambda_{i}\left|1_{i}\right\rangle, \quad \sum_{i=1}^{n}\left|\lambda_{i}\right|^{2}=1 .
$$

In the same way as in the previous section we obtain the relation for concurrence between modes $i$ and $j$

$$
C_{i j}=2\left|\lambda_{i}\right|\left|\lambda_{j}\right|
$$

From the set of equations (17) we can express the probabilities

$$
\left|\lambda_{j}\right|^{2}=\frac{C_{i j} C_{k j}}{2 C_{i k}} \quad i \neq k \neq j .
$$


Therefore, the concurrences $C_{i j}$ have to fulfil the equation

$$
\frac{C_{i j} C_{k j}}{C_{i k}}=\frac{C_{a j} C_{b j}}{C_{a b}},
$$

which has to be valid for all $i, j, k, a, b \in \hat{n}$. Now we fix three indices (modes) $i, j$ and $k$ (naturally $i \neq j \neq k$ ). Therefore, all the concurrences $C_{a b}$ (where $a, b \neq i$ ) can be expressed as a function of concurrences $C_{i a}$ and the concurrence $C_{j k}$

$$
C_{a b}=\frac{C_{i a} C_{i b}}{C_{i j} C_{i k}} C_{j k},
$$

and for $b=j$ we have

$$
C_{a j}=C_{i a} \frac{C_{i k}}{C_{j k}} .
$$

Moreover, the coefficients $\lambda_{i}$ defined by (18) have to fulfil the normalization condition (16). If we use the set of equations (18) the normalization condition takes the form

$$
2=\frac{C_{i j} C_{i k}}{C_{j k}}+\frac{C_{i j} C_{j k}}{C_{i k}}+\sum_{a=1, a \neq i, j}^{n} \frac{C_{i a} C_{j a}}{C_{i j}},
$$

which, with the help of equation (21), results in

$$
2=\frac{C_{i j} C_{i k}}{C_{j k}}+\frac{C_{j k}}{C_{i j} C_{i k}} \sum_{a=1, a \neq i}^{n} C_{i a}^{2} .
$$

Now, it is useful to rewrite equation (23) in the form

$2=x+\frac{K}{x}, \quad$ where $\quad x=\frac{C_{i j} C_{i k}}{C_{j k}} \quad$ and $\quad K=\sum_{a=1, a \neq i}^{n} C_{i a}^{2}$.

The direct consequence of equation (24) is the inequality

$$
0<K=\sum_{a=1, a \neq i}^{n} C_{i a}^{2} \leqslant 1
$$

with two possible solutions of $x$

$$
x=1 \pm \sqrt{1-K} .
$$

The two possible solutions of $C_{j k}$ follow from equations (26) and (24)

$$
C_{j k}=\frac{C_{i j} C_{i k}}{1+\sqrt{1-K}}
$$

or

$$
C_{j k}=\frac{C_{i j} C_{i k}}{1-\sqrt{1-K}} .
$$

Both solutions are physical because they fulfil the condition $0<C_{j k}<1$. With the help of (27) (resp. (28)) equation (20) reads

$$
\begin{aligned}
& C_{a b}=\frac{C_{i a} C_{i b}}{1+\sqrt{1-K}} \\
& \left(\text { resp. } C_{a b}=\frac{C_{i a} C_{i b}}{1-\sqrt{1-K}}\right),
\end{aligned}
$$


and $a, b \neq i$. Finally, we can express the absolute values of the coefficients $\lambda_{i}$ in terms of concurrences $C_{i a}$. From equations (18), (21) and (27) (resp. (28)), we get

$$
\begin{aligned}
\left|\lambda_{j}\right|^{2} & =\frac{C_{1 j}^{2}}{2(1 \pm \sqrt{1-K})}, \quad \text { for } \quad j \neq i \\
\left|\lambda_{i}\right|^{2} & =\frac{1}{2}(1 \pm \sqrt{1-K})
\end{aligned}
$$

Let us now summarize obtained results in the following theorem.

Theorem. Every bipartite entanglement structure $\left\{C_{i j}\right\}$, where $C_{i j} \neq 0$ for all pair of indices $(i, j)$, can be represented by a pure state (16) if and only if for an arbitrary chosen and fixed index (mode) $i$ the condition (25) is fulfilled and for all indices $a$ and $b$ (where $a, b \neq i$ ) the condition (29) (resp. (30)) is fulfilled. If both conditions are fulfilled we can represent this bipartite entanglement structure by a pure state $(16)$, where $\left|\lambda_{j}\right|^{2}=\frac{C_{i j}^{2}}{2(1+\sqrt{1-K})}$, for all $j \neq i$ and $\left|\lambda_{i}\right|^{2}=\frac{1}{2}(1+\sqrt{1-K})$ (resp. $\left|\lambda_{j}\right|^{2}=\frac{C_{i j}^{2}}{2(1-\sqrt{1-K})}$, for all $j \neq i$ and $\left.\left|\lambda_{i}\right|^{2}=\frac{1}{2}(1-\sqrt{1-K})\right)$.

Let us point out two important features. First, it is clear that this bipartite entanglement structure is generated by the set of concurrences $\left\{C_{i j}\right\}$ between the one chosen mode $i$ and the rest of the populated modes. Second, these concurrences can be chosen freely with respect to the restriction rule (25). But these restrictions are actually the well-known conjectured generalized CKW inequalities [13].

It should be emphasized that the form of the entangled state is not unique due to the fact that only the absolute values of the expansion coefficients $\lambda_{i}$ are determined. Also the optical network generating such a state is not unique. For example, if we want to construct a network which generates the desired state when we send a single photon in one particular mode, then we have defined just one row of the transfer matrix of the optical network, while the others remain unspecified. The only additional restriction we impose on the form of the transfer matrix is that it should form a unitary matrix. This freedom of choice of the rest of the transfer matrix can be used to simplify the construction of the optical network with beam splitters and phase shifters using for instance the Zeilinger method [14].

\subsection{Optimization of entanglement distribution in passive networks with one excitation}

In the following, we will denote $C_{i j}=C\left(\rho_{i j}\right)$ for $i \neq j$ and $C_{i i}=0$. For the purpose of optimization we define a function of the total value of the bipartite entanglement for a system described by its density matrix $\rho$

$$
\tau(\rho)=\sum_{(i j)} C_{i j}^{2}
$$

where we sum over all pairs of indices. In the following we will understand under the problem of optimizing the entanglement distribution the maximization of the function (32).

From (15) follows after the summation over the index $j$ the relation (using the normalization condition)

$$
\sum_{j} C_{i j}^{2}=4 P(i)[1-P(i)] .
$$

After the second summation over the index $i$ we get

$$
\sum_{i, j} C_{i j}^{2}=4\left(1-\sum_{i} P(i)^{2}\right),
$$


and all pairs of indices $(i, j)$ appeared in the sum twice. Therefore, we take only one-half to arrive finally at

$$
\sum_{(i, j)} C_{i j}^{2}=2\left(1-\sum_{i} P(i)^{2}\right) .
$$

We have to maximize (35) with the additional condition $\sum_{i} P(i)=1$. It is not difficult to show that

$$
0 \leqslant \sum_{(i, j)} C_{i j}^{2} \leqslant 2\left(1-\frac{1}{N}\right)=\frac{N(N-1)}{2}\left(\frac{2}{N}\right)^{2} .
$$

Thus, the maximum attainable value of $\tau(\rho)$ is

$$
\tau_{\max }(N)=2\left(1-\frac{1}{N}\right)
$$

and is achieved for $P(i)=\frac{1}{N}$. In this case, all pairs are entangled alike and the single pair entanglement has the value

$$
C_{i j}=\frac{2}{N} \text {. }
$$

Similar results have been obtained by Li et al [16]. We can compare the obtained optimal value with a result which were published by Koashi et al [15]. They studied completely symmetric states (with respect to all permutation of qubits) of $N$ qubits and found out that the maximum value of the concurrence between arbitrary qubits is the same, i.e.

$$
C=\frac{2}{N}
$$

It is important to realize that the identification of an optimal network is closely related to the given input state. For example, we wish to construct a network which distributes in the optimal way the entanglement for the case, when we send a single excitation in one mode (no superposition between the input modes). In this case, the condition $P(i)=\frac{1}{N}$ for all $i \in \hat{N}$ is equivalent to the condition $\left|U_{m n}\right|^{2}=\frac{1}{N}$ for all $m, n \in \hat{N}$. This condition fulfils, for example, the discrete Fourier transform in all its possible forms $[17,18]$.

\subsection{Balanced Ising-type network}

In the previous sections, we have derived results on entanglement which are valid for all passive networks. In the following, we use these results for the balanced Ising network.

We will concentrate on two different situations. First, we study the case when we are sending one excitation in one of the modes (no superposition between the modes at the input) to the infinite network. Because the network is infinite and translation invariant with respect to the inputs it does not matter through which of the inputs the excitation is entering the network.

The propagation of the probability distribution of a single excitation in the network dependent on the traversed motifs is shown in figure 2. The plot clearly illustrates the unusual behaviour, when one of the direction of spreading is preferred [3]. In the next graph (figure 3), the propagation of the bipartite entanglement along particular motifs is shown. In agreement with (15) we observe that the propagation of the bipartite entanglement also prefers the same direction, the concurrence exhibits similar oscillatory behaviour like the photon number distribution. 


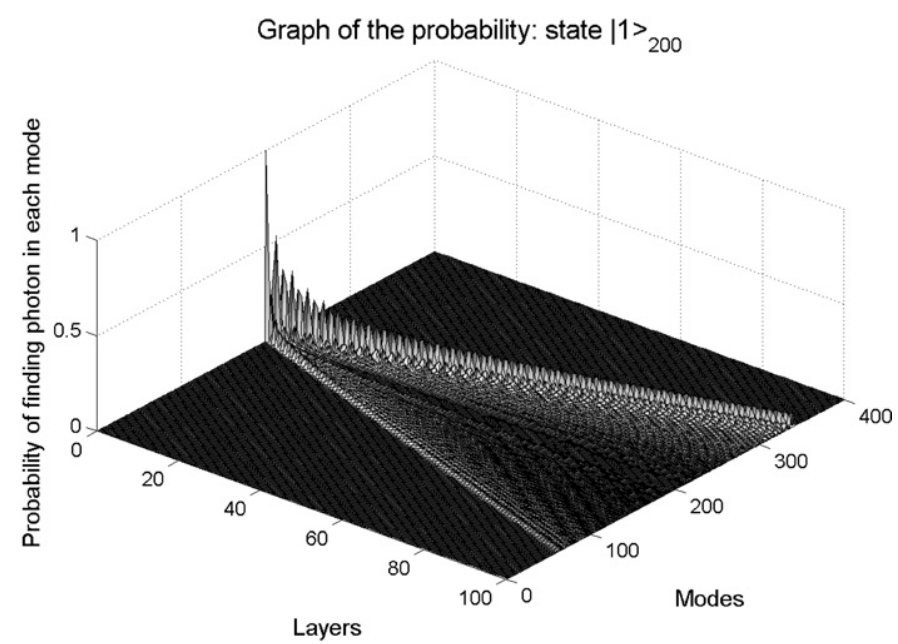

Figure 2. The probability of finding the excitation in an arbitrary mode and motif. The excitation starts its propagation in the input state $\left|1_{200}\right\rangle$ and is numerically simulated for 100 motifs.

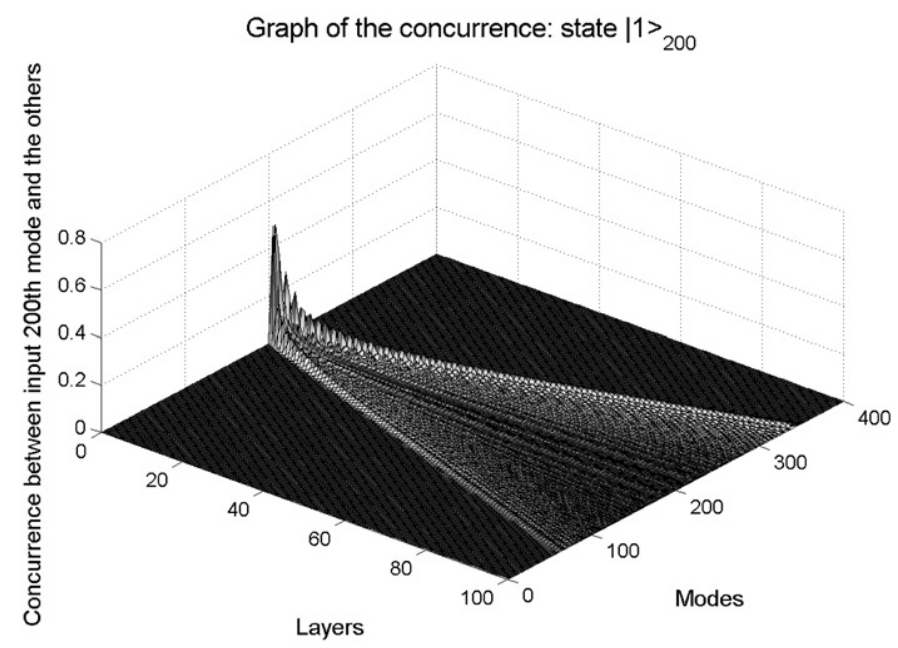

Figure 3. Graph of the concurrence between mode 200 and the others dependent on the number of motifs traversed. The excitation starts its propagation in the input state $\left|1_{200}\right\rangle$ and is numerically simulated up to 100 motifs.

\subsection{Balanced Ising-type network with phase errors}

The second problem we wish to address is the evolution of the concurrence in the case we have phase errors in the system. The randomization of the phases before the excitation enters the next layer can be performed in two ways. The first is to perturb the phases after each layer independently and this leads to a Gaussian probability distribution after averaging. Due to the relation between the probability distribution and the concurrence (15) it has a similar effect to the entanglement distribution. When the once generated phase perturbation is kept through the evolution and we average over many realization we arrive at an exponential localization in 


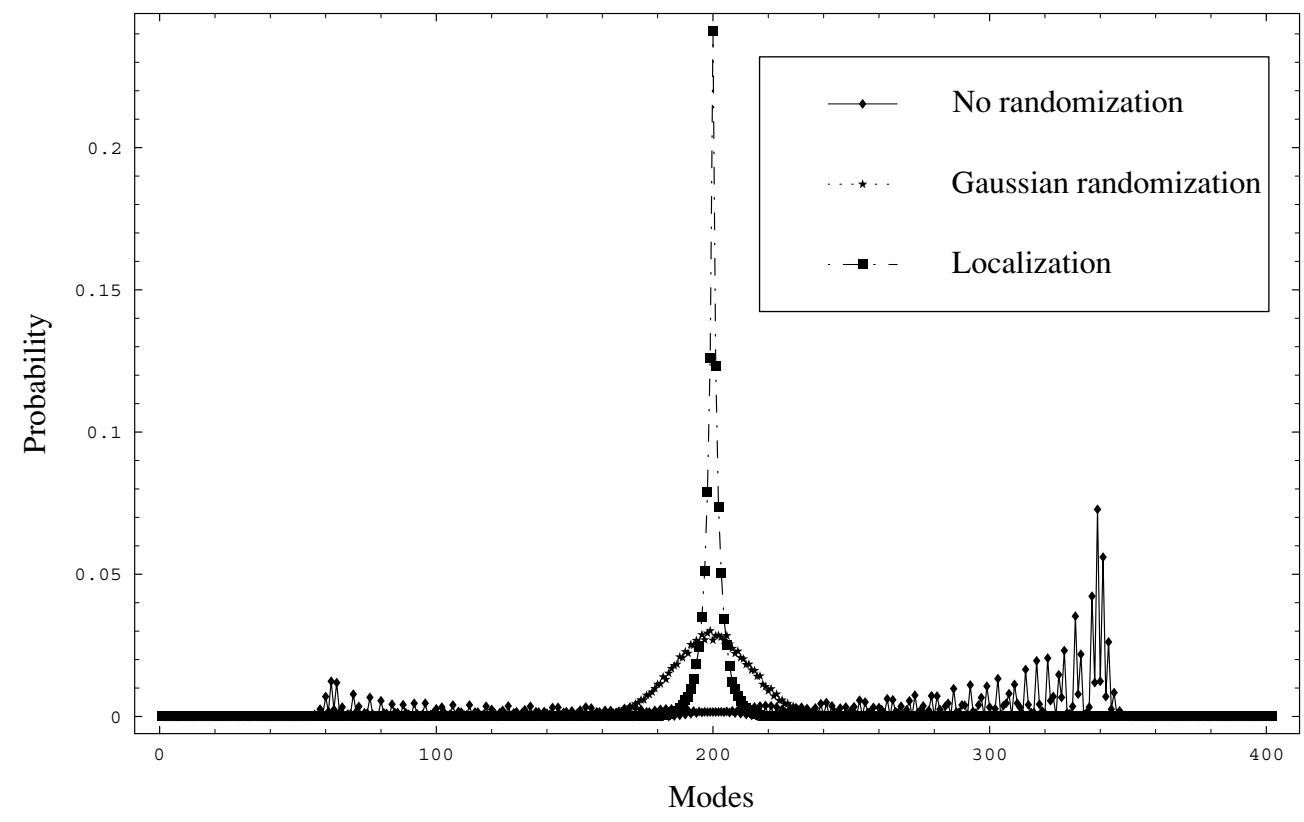

Figure 4. The probability distributions of detecting a photon after 100 motifs applied. The excitation starts its propagation in the input state $\left|1_{200}\right\rangle$ and is numerically simulated up to 100 motifs.

the probability distribution and consequently in the entanglement distribution. This result is in accordance with the results obtained for single-photon evolution in the network [4].

To illustrate how the random phases influence the probability distribution we plot in figure 4 the result for the situation when the photon enters the network in the state $\left|1_{200}\right\rangle$ and after traversing through 100 motifs. For the randomization we have averaged the probability distribution over 1000 runs.

Let us now analyse how the two different approaches of the randomization influence the total amount of the entanglement in the network. The total amount of the entanglement is measured by the sum of squared concurrences (32). For every run we have calculated the function (32) at each motif and then averaged over 1000 runs. For comparison we plot in figure 5 the dependence of (32) for the optimal value, the unperturbed value and both types of randomization. Because at the $M$ th motif $4 M$ modes are populated and hence entangled, we take as the optimal value $\tau_{\max }(4 M)=2\left(1-\frac{1}{4 M}\right)$, see (37).

The plot indicates that the overall unperturbed value $\tau(\rho)$ is approaching the optimum value for a large number of traversed motifs. Even though, in the beginning, the change of $\tau(\rho)$ is oscillatory, it levels out for large numbers of motifs.

The randomization leading to the Gaussian probability distribution does not decrease the entanglement dramatically and numerical simulations show that the overall value is still approaching the optimal value for a large number of steps. On the other hand, the second type of randomization leading to the exponential localization has a significant effect. The overall averaged entanglement in the network is reduced dramatically, it does not converge to the optimal value. Thus, the two different implementations of the random phase have a completely different effect on the average entanglement distribution in the network. 


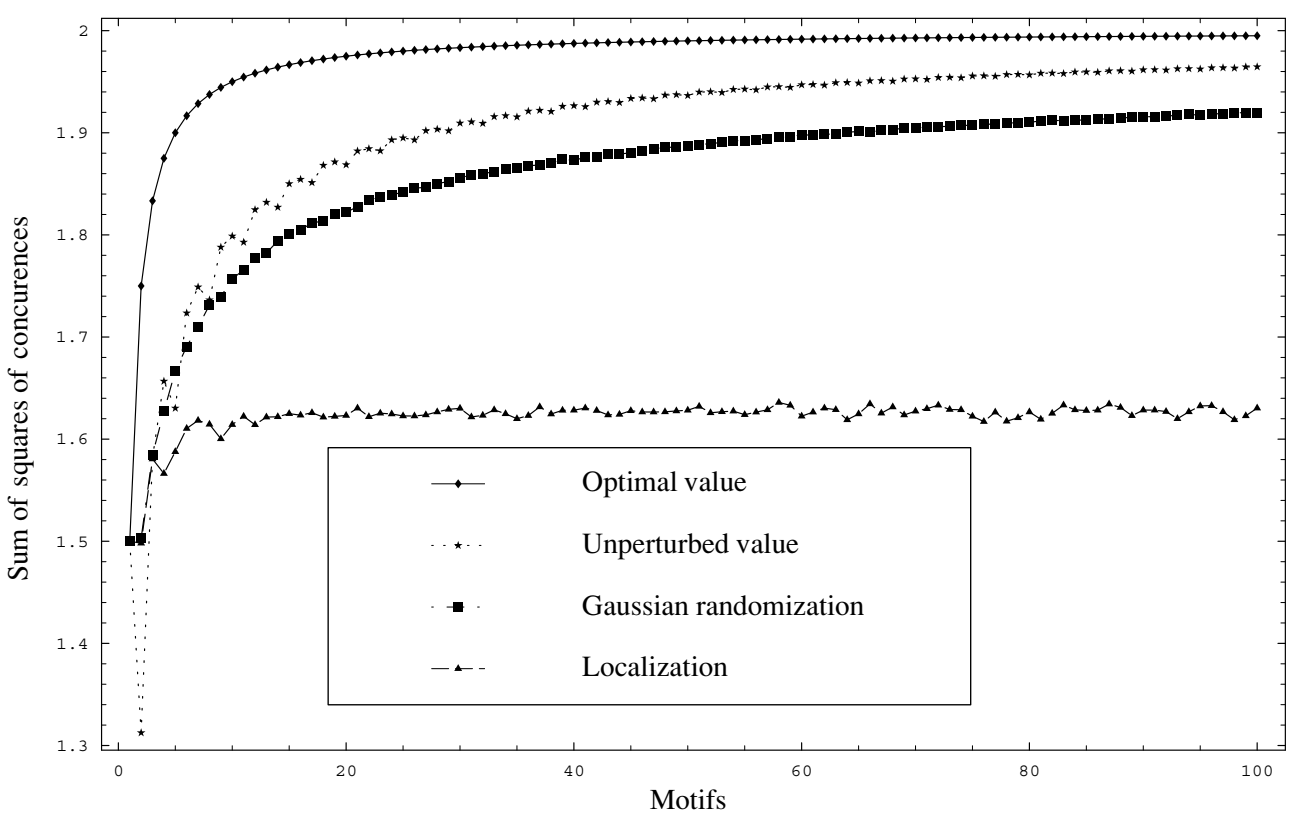

Figure 5. Graph of the function (32) for the optimal value, balanced Ising network with no phase errors and two different implementations of phase errors. The excitation starts its propagation in state $\left|1_{200}\right\rangle$ and is numerically simulated up to 100 motifs applied.

\section{Entanglement distribution in passive networks with two-photon input}

The quantification of the entanglement in the case of two excitations in the network is a bit more involved than in the case of a single excitation. A closed expression for the concurrence quantifying the amount of entanglement can be derived for the particular case when the total probability of detecting two photons at any of the outputs goes to zero for large network (large number of traversed motifs). In this case, we can limit ourselves to the description of quantum state propagation for states having form $\left|0_{i}\right\rangle\left|1_{j}\right\rangle \cdots\left|1_{k}\right\rangle \cdots\left|0_{l}\right\rangle(j \neq k)$, i.e. in each of the modes at most one excitation is present. In this case, the application of the concurrence concept is completely legitimate and reflects properly the entanglement distribution within the network.

The requirement on the extinction of the two-photon probabilities is for instance satisfied for the nearest-neighbour Ising model (or the Fourier transform acting on all the inputs) for two-photon inputs via one input mode. When all the single-photon output probabilities scale typically as $1 / N$ the individual two-photon probabilities at one output will scale as $1 / N^{2}$. From this follows that the total probability that two photons emerge from the network via any of the outputs scales as $1 / N$ and hence tends to zero for large enough networks. In the following, we will neglect the probability of the double excitations in one mode.

Let us choose an arbitrary two-particle input state

$$
\left|\psi_{\mathrm{in}}\right\rangle=\sum_{(i j)} \alpha_{i j} a_{i}^{\dagger} a_{j}^{\dagger}|0\rangle+\frac{1}{\sqrt{2}} \sum_{i=1}^{N} \alpha_{i} a_{i}^{\dagger 2}|0\rangle=\sum_{(i j)} \alpha_{i j}\left|1_{i} 1_{j}\right\rangle+\sum_{i=1}^{N} \alpha_{i}\left|2_{i}\right\rangle
$$


where in the first term the summation is done over all $\left(\begin{array}{l}n \\ 2\end{array}\right)$ pairs of different indices $i, j$. Then the output state has the form

$$
\left|\psi_{\text {out }}\right\rangle=\sum_{k, l=1}^{N}\left\{\sum_{(i j)} U_{k i} U_{l j} \alpha_{i j}+\sum_{i=1}^{N} \frac{1}{\sqrt{2}} U_{k i} U_{l i} \alpha_{i}\right\} b_{k}^{\dagger} b_{l}^{\dagger}|0\rangle .
$$

To simplify the lengthy notation we denote

$$
\begin{aligned}
& \beta_{k l}=\sum_{(i j)} 2 U_{k i} U_{l j} \alpha_{i j}+\sum_{i=1}^{N} \sqrt{2} U_{k i} U_{l i} \alpha_{i} \\
& \beta_{k}=\sum_{(i j)} \sqrt{2} U_{k i} U_{k j} \alpha_{i j}+\sum_{i=1}^{N} U_{k i} U_{k i} \alpha_{i},
\end{aligned}
$$

using these coefficients we can rewrite the output state in the form

$$
\left|\psi_{\text {out }}\right\rangle=\sum_{(k l)} \beta_{k l}\left|1_{k} 1_{l}\right\rangle+\sum_{k=1}^{N} \beta_{k}\left|2_{k}\right\rangle
$$

To fulfil the condition that the two-photon excitations must vanish we have to put $\beta_{k}=0$ for all modes $k$. The renormalized output state then has the form

$$
\left|\psi_{\text {out }}\right\rangle=\frac{1}{K} \sum_{(k l)} \beta_{k l}\left|1_{k} 1_{l}\right\rangle
$$

where $K$ is determined by

$$
K^{2}=\sum_{(k l)}\left|\beta_{k l}\right|^{2}
$$

The reduced density operator of two fixed modes $a$ and $b$ is easily evaluated, the result is given by

$$
\rho_{a b}=\left(\begin{array}{cccc}
\left|\beta_{a b}\right|^{2} & 0 & 0 & 0 \\
0 & \sum_{k \neq a, b}\left|\beta_{a k}\right|^{2} & \sum_{k \neq a, b} \beta_{a k} \beta_{b k}^{*} & 0 \\
0 & \sum_{k \neq a, b} \beta_{a k}^{*} \beta_{b k} & \sum_{k \neq a, b}\left|\beta_{b k}\right|^{2} & 0 \\
0 & 0 & 0 & \sum_{(k l) \neq(a b)}\left|\beta_{k l}\right|^{2}
\end{array}\right),
$$

and the normalization constant was absorbed into the definition of $\beta_{k l}$.

To evaluate the degree of entanglement between two chosen modes we have to determine the eigenvalues of the matrix (11) associated with the reduced density matrix (46). For the eigenvalues we obtain the following expressions:

$$
\begin{aligned}
& \lambda_{1,2}=\left(\sqrt{\sum_{k \neq a, b}\left|\beta_{a k}\right|^{2} \sum_{k \neq a, b}\left|\beta_{b k}\right|^{2}} \pm\left|\sum_{k \neq m, n} \beta_{a k} \beta_{b k}^{*}\right|\right)^{2} \\
& \lambda_{3,4}=\left(\left|\beta_{a b}\right| \sqrt{\sum_{(k, l) \neq a, b}\left|\beta_{k l}\right|^{2}}\right)^{2} .
\end{aligned}
$$


A necessary condition for the existence of entanglement between the modes $a, b$ is that the eigenvalue $\lambda_{1}$ is larger than the eigenvalues $\lambda_{3,4}$. Using these eigenvalues and the expression for the concurrence (12), we obtain

$$
C_{a b}=2 \max \left(\left|\sum_{k \neq a, b} \beta_{a k} \beta_{b k}^{*}\right|-\left|\beta_{a b}\right| \sqrt{\sum_{(k, l) \neq a, b}\left|\beta_{k l}\right|^{2}}, 0\right) .
$$

Let us emphasize that the obtained result holds generally for any two-particle state superpositions of the form (44). The expressions for the concurrence differ in their characters from the simpler type valid for single-photon concurrence. First of all the concurrence is dependent not only on the absolute value of the expansion coefficients but also on their relative phases. The single-photon concurrence was dependent only on their amplitude.

The expression for the concurrence can be written for the special type of state with two photons in the same input mode

$$
\left|\psi_{\text {in }}\right\rangle=\frac{1}{\sqrt{2}} \hat{a}_{i}^{\dagger 2}|0\rangle
$$

in a simplified way. With the help of the definition (42) and the fact that $\beta_{i}=0$ for all modes $i$ we obtain the following form of the eigenvalues:

$$
\begin{aligned}
& \lambda_{1}=2 \sqrt{\left(\sum_{k \neq a, b}\left|\beta_{a k}\right|^{2}\right)\left(\sum_{l \neq a, b}\left|\beta_{b l}\right|^{2}\right)}, \\
& \lambda_{2,3}=\left|\beta_{a b}\right| \sqrt{\sum_{(k l) \neq(a b)}\left|\beta_{k l}\right|^{2}}, \quad \lambda_{4}=0 .
\end{aligned}
$$

We now define the following probabilities of detecting the photons:

- $P\left({ }^{i j}\right)$ : the probability of detecting the photons in both modes $i$ and $j$,

- $P\left({ }_{i j}\right)$ : the probability that no photons will be detected in modes $i$ and $j$,

- $P\left(\begin{array}{l}i \\ j\end{array}\right)$ : the probability of detecting one photon in mode $i$ and no photon in mode $j$,

$$
\begin{aligned}
& P\left(\begin{array}{c}
i j \\
\end{array}\right)=\left|\left\langle 1_{i} 1_{j} \mid \psi_{\text {out }}\right\rangle\right|^{2}=\left\langle 11\left|\rho_{i j}\right| 11\right\rangle=\left|\beta_{i j}\right|^{2} \\
& P\left(\begin{array}{c} 
\\
i j
\end{array}\right)=\sum_{(k l) \neq(i j)}\left|\left\langle 1_{k} 1_{l} \mid \psi_{\text {out }}\right\rangle\right|^{2}=\left\langle 00\left|\rho_{i j}\right| 00\right\rangle=\sum_{(k l) \neq(i j)}\left|\beta_{k l}\right|^{2} \\
& P\left(\begin{array}{c}
i \\
j
\end{array}\right)=\sum_{k \neq i, j}\left|\left\langle 1_{i} 1_{k} \mid \psi_{\text {out }}\right\rangle\right|^{2}=\left\langle 10\left|\rho_{i j}\right| 10\right\rangle=\sum_{k \neq i, j}\left|\beta_{i k}\right|^{2} .
\end{aligned}
$$

With the help of these probabilities we can rewrite the eigenvalues (51) and express the concurrence of modes $a$ and $b$ in a simple form

$$
C_{a b}=2\left[\sqrt{P\left(\begin{array}{l}
a \\
b
\end{array}\right) P\left(\begin{array}{l}
b \\
a
\end{array}\right)}-\sqrt{P\left({ }_{a b}\right) P\left(\begin{array}{l}
a b \\
\end{array}\right)}\right] .
$$




\subsection{Balanced Ising-type network}

Let us now specify the result (53) for the case of the balanced Ising-type network. In this case, we can describe the propagation of the two photons in the network using the results obtained for the propagation of a single photon studied in section 3 . We can rewrite formula (53) using the single-photon probabilities (10). We will denote the probability of detecting the photon (entering the network via the mode $i$ ) in the output mode $k$ as

$$
P_{i}(k)=\left|U_{k i}\right|^{2} \text {. }
$$

In our case, we have $\alpha_{m}=\delta_{m i}, \alpha_{i j}=0$. If we put these coefficients into the relations (45) and (52), using the definition (10), we obtain

$$
\begin{aligned}
& P\left(\begin{array}{c}
m n \\
)
\end{array}\right)=\frac{2 P_{i}(m) P_{i}(n)}{1-\sum_{k=1}^{N} P_{i}(k)^{2}} \\
& P\left(\begin{array}{c} 
\\
m n
\end{array}\right)=\frac{\left(1-\sum_{k=1}^{N} P_{i}(k)^{2}\right)-2 P_{i}(m)-2 P_{i}(n)+2 P_{i}(m)^{2}+2 P_{i}(n)^{2}+2 P_{i}(m) P_{i}(n)}{1-\sum_{k=1}^{N} P_{i}(k)^{2}} \\
& P\left(\begin{array}{c}
m \\
n
\end{array}\right)=\frac{2 P_{i}(m)\left(1-P_{i}(m)-P_{i}(n)\right)}{1-\sum_{k=1}^{N} P_{i}(k)^{2}} .
\end{aligned}
$$

Using these results we can rewrite formula (53) in the form

$$
\begin{aligned}
C_{a b}= & \frac{4 \sqrt{P_{i}(a) P_{i}(b)}}{1-\sum_{k=1}^{N} P_{i}(k)^{2}}\left[\left(1-P_{i}(a)-P_{i}(b)\right)\right. \\
& \left.-\sqrt{\frac{\left(1-\sum_{k=1}^{N} P_{i}(k)^{2}\right)}{2}-P_{i}(a)-P_{i}(b)+P_{i}(a)^{2}+P_{i}(b)^{2}+P_{i}(a) P_{i}(b)}\right] .
\end{aligned}
$$

The way how the entanglement evolves in the Ising-type network is shown in figure 6 . The plot starts after the photons have passed through 30 motifs and shows the evolution up to 100 motifs, this is due to the fact that for small number of motifs passed we cannot omit the two-photon excitations. After the photons pass through 30 motifs the total probability of detecting two excitations in one mode is smaller than $5 \%$ so we can neglect the two-photon contributions.

Let us analyse the total amount of entanglement in the network, which we measure by the function (32). In contrast to the single-photon case we cannot give a simple formula for this function due to the complicated form of the bipartite concurrence (49). Thus, the maximization of the overall entanglement in the two-photon case is much more difficult compared to the single-photon case. We evaluated numerically the function $\tau(N)$ for the case of balanced Ising network with two photons entering in the state (50). We compare this value with the value for the uniform distribution (note that we have $4 N$ modes populated after $N$ motifs applied), for which we obtain

$$
C_{i j}=\frac{(4 N-2-\sqrt{(2 N-1)(4 N-3)})}{N(4 N-1)}, \quad i \neq j,
$$

i.e., all populated modes are equally entangled. The overall entanglement has the value

$$
\tau_{1}(N)=\frac{2(4 N-2-\sqrt{(2 N-1)(4 N-3)})^{2}}{N(4 N-1)} .
$$

The limit value of this function is

$$
\lim _{N \rightarrow+\infty} \tau_{1}(N)=12-8 \sqrt{2} \approx 0.686,
$$




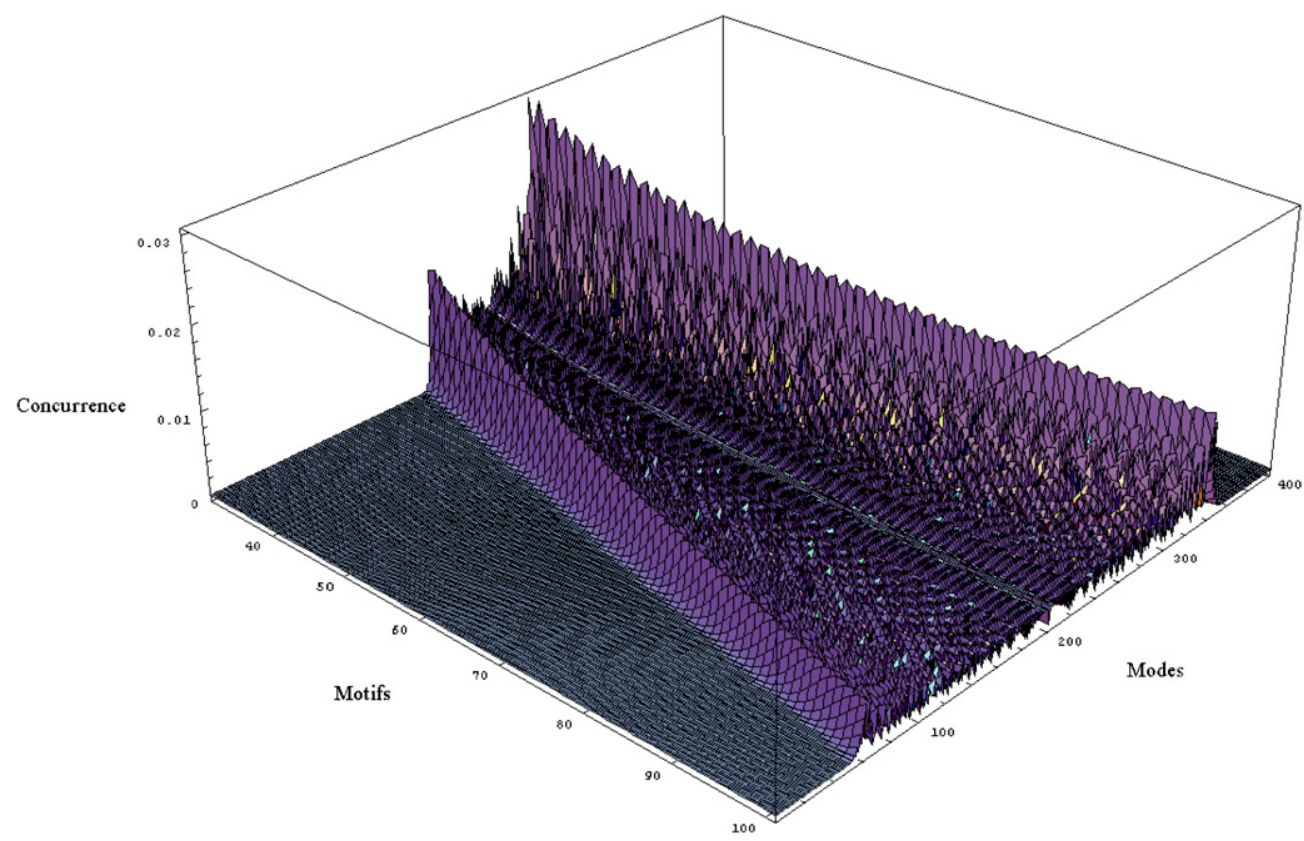

Figure 6. Graph of the concurrence between mode 200 and the others dependent on the number of motifs traversed. The two excitations start the propagation in the input state $\left|2_{200}\right\rangle$ and are numerically simulated up to 100 motifs.

thus we can see that the overall entanglement in the network with two excitations is reduced dramatically compared to the network with single excitation, where the limit value was 2 . Figure 7 illustrates how the total concurrences evolve with the number of motifs passed $N$. The numerical simulations suggest that they converge, similar to the case of the single-photon initial state.

For the case of the single-photon input the uniform distribution was the optimal one concerning the overall entanglement. To show that this is no more valid for the case of two photons, we consider the following factorized two-photon state of the form

$$
|\psi\rangle=\frac{1}{\sqrt{2 N}} \sum_{i=1}^{2 N}\left|1_{2 i}\right\rangle \otimes \frac{1}{\sqrt{2 N}} \sum_{i=0}^{2 N-1}\left|1_{2 i+1}\right\rangle .
$$

In this case, the two photons are completely independent, one is uniformly distributed over odd modes and the second one over even modes. The bipartite entanglement structure has the following form:

$$
\begin{array}{ll}
C_{2 i, 2 j+1}=C_{2 j+1,2 i}=0, & i=1, \ldots, N, \quad j=0, \ldots, N-1, \\
C_{2 i, 2 j}=C_{2 i+1,2 j+1}=\frac{1}{N}, & i \neq j,
\end{array}
$$

i.e., only odd or even modes are equally entangled together, odd modes stay unentangled with the even modes and vice versa. Thus, the sum of squared concurrences can be decomposed into the sum over odd and even pairs of indices and equals to

$$
\tau_{2}(N)=\sum_{k l} C_{(a b)}^{2}=4\left(1-\frac{1}{2 N}\right),
$$




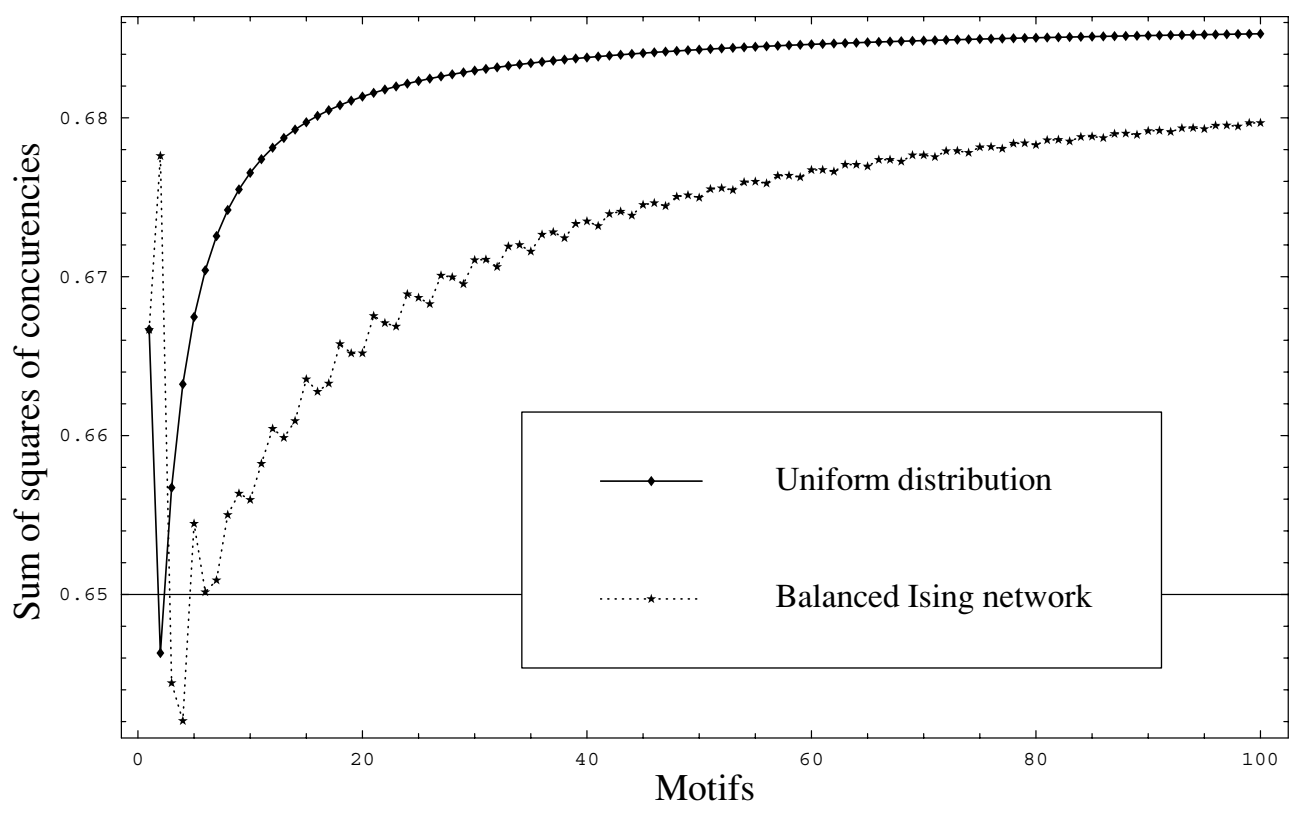

Figure 7. Graph of sum of squared concurrences dependent on the number of motifs traversed. The two excitations start the propagation in the input state $\left|2_{200}\right\rangle$ and are numerically simulated up to 100 motifs.

which reaches asymptotically the value 4 . Compared to this value the value for the uniformly distributed states (58) is very moderate.

The very moderate amount of entanglement for the uniformly distributed state is a price that has to be paid to the additional high symmetry of the bipartite entanglement between the modes. For the uniformly distributed state with the distribution (58) all the bipartite concurrences are the same. When we relax this condition states can be found for which the total amount of entanglement will increase.

\section{Conclusions}

We studied the evolution of single- and two-photon states in passive optical network, in particular in passive networks realizing nearest-neighbour interaction (Ising model). We have shown that how the mutual entanglement changes and that for special initial states the maximum attainable entanglement can be almost reached with the Ising-type passive network. In addition, we derived closed-form expression for the degree of entanglement for general passive networks and proved what the maximum entanglement for single-photon input is. The influence of randomization on the average degree of entanglement was discussed for single-photon input states. For two-photon inputs we derived closed-form expressions for the bipartite entanglement for a broad class of states (realized for instance by the Isingtype networks). We have shown that the total amount of entanglement reached in Ising-type networks is rather moderate compared to the maximum obtainable value for special (exhibiting a certain symmetry) two-photon states. 


\section{Acknowledgments}

This work was supported by the Czech and Hungarian Ministries of Education under contract no. CZ-5/03 (Hungarian-Czech Scientific Cooperation), by the GAČR 202/04/2101, EU QUELE and by the Hungarian Scientific Research Fund (contract no. T43287).

\section{References}

[1] Ou Z, Hong C K and Mandel L 1987 Opt. Commun. 63118

[2] Barnett S M, Chefles A and Jex I 2003 Phys. Lett. A 307189

[3] Kempe J 2003 Contemp. Phys. 444

[4] Törma P, Jex I and Schleich W P 2002 Phys. Rev. A 65052110

[5] Shepelyansky D 1994 Phys. Rev. Lett. 732607

[6] Song P H and von Oppen F 1999 Phys. Rev. B 5946

[7] Törma P 1998 Phys. Rev. Lett. 812185

[8] Toda M, Kubo R and Saito N 1995 Statistical Physics I 2nd edn (Berlin: Springer)

[9] Törma P and Jex I 1997 J. Mod. Opt. 44875

[10] Törma P and Jex I 1999 J. Opt. B 18

[11] Hill S and Wootters W K 1997 Phys. Rev. Lett. 785022

[12] Wootters W K 1998 Phys. Rev Lett. 802245

[13] Coffman V, Kundu J and Wootters W K 2000 Phys. Rev A 61052306

[14] Reck M, Zeilinger A, Bernstein H J and Bertani P 1994 Phys. Rev. Lett. 7358

[15] Koashi M, Bužek V and Imoto N 2000 Phys. Rev. A 62050302

[16] Haibin Li, Wang Xiaoguang and Bambi Hu 2005 J. Phys. A: Math. Gen. 3710665

[17] Dita P 2004 J. Phys. A: Math. Gen. 375355

[18] Vourdas A and Dunningham J A 2005 Phys. Rev. A 71013809 